\title{
Correctly performed nasal swabs
}

\author{
Konstantin Zedtwitz-Liebenstein ${ }^{1}$
}

Received: 10 February 2021 / Accepted: 15 March 2021 / Published online: 26 March 2021

(c) Springer-Verlag GmbH Germany, part of Springer Nature 2021

\begin{abstract}
Background As the incidence of new cases of coronavirus disease increased exponentially, the use of viral swabs to collect nasopharyngeal specimens are increasing drastically. Therefore, healthcare workers military staff and uneducated nonprofessional's were ordered to make this swabs. Subsequently case reports reported about basal skull perforation, cerebrospinal fluid fistula and injury due to an incorrect technique.

Methods Search of the literature.

Results Only in $44 \%$ of the videos (Youtube) nasal swabs were correctly performed. Due to an false technique biological sampling resulted in false-negative COVID-19 tests.

Conclusion Although professional societies started to report about this unacceptable situation, no publication reported about this health endangerment. In this time of overwhelming information and diversity of opinions, it is necessary to report about this in the hope, all media and TV reports will follow this article to show correctly performed nasal swabs to reduce falsenegative COVID-19 tests and injury.
\end{abstract}

Keywords Nasal swab $\cdot$ Injury $\cdot$ Endangerment $\cdot$ False-negative test

The COVID-19 infection can be diagnosed from upper and lower respiratory sources including the oropharynx, nasopharynx, sputum, and bronchial fluid. It appears to be extremely important to properly collect nasopharyngeal swabs reaching the posterior rhinopharyngeal tonsil region. To correctly perform the nasal swab, the patient must be seated with the back of their head against the headrest. The swab is inserted in the nose horizontally, along an imaginary line between the nostril and the ear (tragus). Upon reaching the posterior wall of the nasopharynx, it is not necessary to rotate the swab in place according to a study, before gently removing the swab [1]. The investigator has to be careful not to insert the swab in an upwards direction or limit the sampling to only the anterior portion of the nasal cavity as this would reduce the probability of taking a significant amount of viral RNA (Fig. 1).

In a study, suboptimal biological sampling resulted in false-negative COVID-19 tests [droplet digital polymerase chain reaction (ddPCR)]. The results showed the importance

Konstantin Zedtwitz-Liebenstein

konstantin.zedtwitz-liebenstein@gesundheitsverbund.at

$1 \quad$ Head Office of Vienna Hospital Association, Thomas Klestil

Platz 7, 1030 Vienna, Austria of proper training and technique in the collection of nasopharyngeal specimens [2]. For this procedure, there still exist different instructions from professional societies and publications [3, 4]. The Centers for Disease Control and Prevention (CDC) recommend tilting patient's head back in $70^{\circ}$ [5]. Such an angle of inclination complicates to perform a correct nasal swab by an uneducated health care worker or a nonprofessional (Fig. 2).

As the incidence of new cases of coronavirus disease increased exponentially, the use of viral swabs to collect nasopharyngeal specimens were increasing drastically and therefore healthcare workers military staff and uneducated nonprofessional's were ordered to make these swabs. Subsequently, case reports reported about basal skull perforation, cerebrospinal fluid fistula and injury due to an incorrect technique $[6,7]$.

An anatomically accurate three-dimensional (3D) printed model of the nose was used during training sessions for healthcare workers for nasopharyngeal swabs. This enhanced realistic, efficient, and safe nasopharyngeal swab training, and helped to ensure that swab results are as 


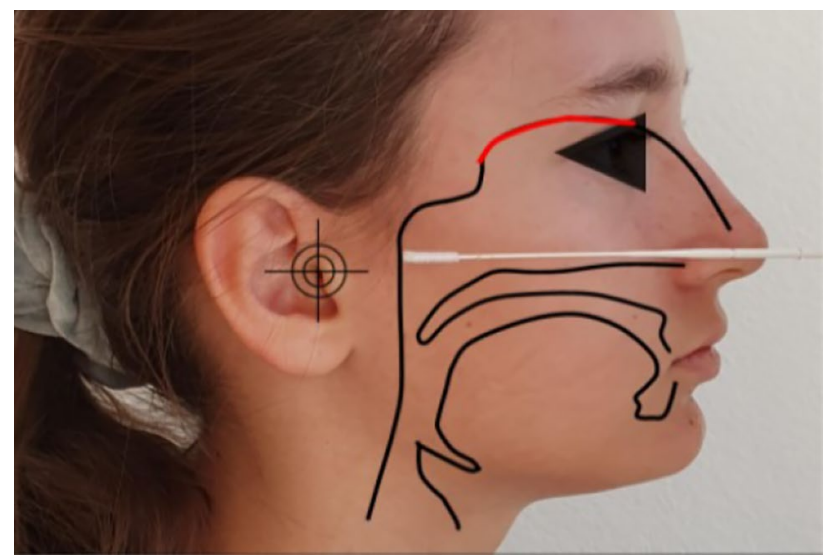

Fig. 1 The swab should be inserted in the nose horizontally along an imaginary line between the nostril and the ear (tragus). The investigator has to be careful not to insert the swab in an upwards direction or limit the sampling to only the anterior portion of the nasal cavity

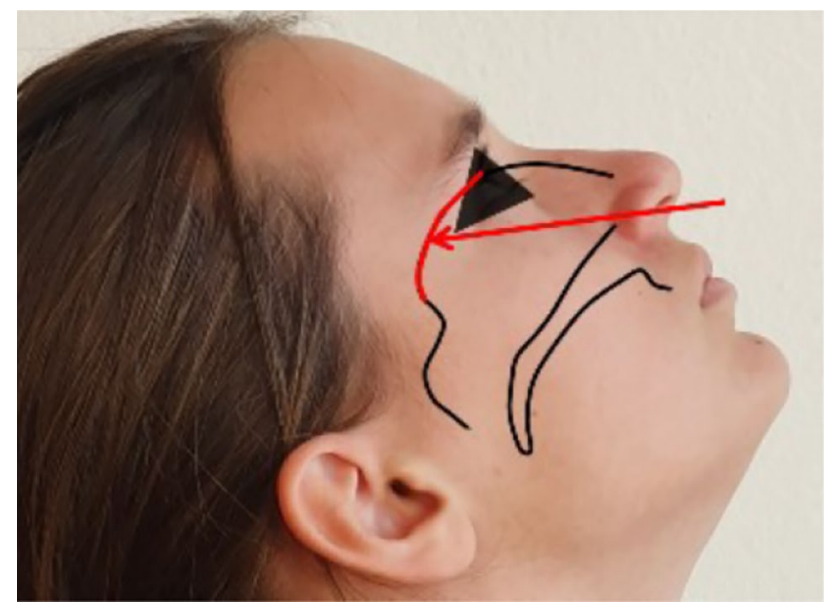

Fig. 2 The Centers for Disease Control and Prevention (CDC) recommend tilting patient's head back in $70^{\circ}$. Such an angle of inclination complicate to perform a correct nasal swab by an uneducated health care worker. If the swab is inserted in the nose, horizontally basal skull perforation and injuries due to an incorrect technique will happen

accurate as possible [8]. Implementation of nasal anatomy education and nasopharyngeal swab technique can improve accurate COVID testing [9].

In a study, three blinded rhinologists rated 126 videos (YouTube) for accuracy of swab angle and depth. Only in $44 \%$ of the videos nasal swab were correctly performed associated with the swab operator identifying as a medical professional (96\%) [10].

Although professional societies started to report about this unacceptable situation, no publication reported about this health endangerment. In this time of overwhelming information and diversity of opinions, it is necessary to report about this in the hope, all media and TV reports will follow this article to show correctly performed nasal swabs to reduce false-negative COVID-19 tests and injury.

Funding The author received no specific commercial funding.

\section{Declarations}

Conflict of interest The authors declare that they have no conflict of interest.

Consent for publication The author worked at medical university Vienna at the department of infectious diseases and published in the field of virus diseases.

\section{References}

1. Kinloch NN, Shahid A, Ritchie G, Dong W, Lawson T, Montaner JSG, Romney MG, Stefanovic A, Matic N, Brumme CJ, Lowe $\mathrm{CF}$, Brumme ZL, Leung V. Evaluation of nasopharyngeal swab collection techniques for nucleic acid recovery and participant experience: recommendations for COVID-19 diagnostics. Open Forum Infect Dis. 2020;7:1-6.

2. Kinloch NN, Ritchie G, Brumme CJ, Dong W, Dong W, Lawson T, Jones RB, Montaner JSG, Leung V, Romney MG, Stefanovic A, Matic N, Lowe CF, Brumme ZL. Suboptimal biological sampling as a probable cause of false-negative COVID-19 diagnostic test results. J Infect Dis. 2020;222:899-902.

3. Petruzzi G, De Virgilio A, Pichi B, Mazzola F, et al. COVID-19: nasal and oropharyngeal swab. Head Neck. 2020;42:1303-4.

4. Higgins TS, Wu AW, Ting JY. SARS-CoV-2 nasopharyngeal swab testing-false-negative results from a pervasive anatomical misconception. JAMA Otolaryngol Head Neck Surg. 2020;146:993.

5. CDC Centers for Disease Control and Prevention-CDC 24/7: Saving Lives, Protecting PeopleTM Interim guidelines for collecting, handling, and testing clinical specimens from persons for Coronavirus Disease 2019 (COVID-19) https://www.cdc.gov/ coronavirus/2019-ncov/lab/guidelines-clinical-specimens.html

6. Mughal Z, Luff E, Okonkwo O, Hall CEJ. Test, test, test-a complication of testing for coronavirus disease 2019 with nasal swabs. J Laryngol Otol. 2020;134:646-9.

7. Sullivan CB, Schwalje AT, Jensen M, Li L, Dlouhy BJ, Greenlee JD, Walsh JE. Cerebrospinal fluid leak after nasal swab testing for Coronavirus Disease 2019. JAMA Otolaryngol Head Neck Surg. 2020;146:1179-81.

8. Chee J, Lin X, Lim WS, et al. Using 3D-printed nose models in nasopharyngeal swab training. Oral Oncol. 2020;8:105033.

9. Li L, Shim T, Zapanta PE. Optimization of COVID-19 testing accuracy with nasal anatomy education. Am J Otolaryngol. 2021;42:102777.

10. Itamura K, Wu A, Illing E, Ting J, Higgins T (2021) YouTube videos demonstrating the nasopharyngeal swab technique for SARSCoV-2 specimen collection: content analysis. JMIR Public Health Surveill 7(1):e24220 